\title{
Selection of oncoplastic surgical technique in Asian breast cancer patients
}

\author{
Eui Sun Shin, Hyo In Kim, Seung Yong Song, Dae Hyun Lew, Dong Won Lee \\ Institute for Human Tissue Restoration, Department of Plastic and Reconstructive Surgery, Severance Hospital, Yonsei University College of \\ Medicine, Seoul, Korea
}

Background Oncoplastic surgery is being increasingly performed in Korean women; however, unlike Westerners, Korean women usually have small to moderate-sized breasts. To achieve better outcomes in reconstructed breasts, several factors should be considered to determine the optimal surgical method.

Methods A total of 108 patients who underwent oncoplastic surgery from January 2013 to December 2016 were retrospectively investigated. We used various methods, including glandular tissue reshaping, latissimus dorsi (LD) flap transposition, and reduction oncoplasty, to restore the breast volume and symmetry.

Results The mean weight of the tumor specimens was $40.46 \mathrm{~g}$, and the ratio of the tumor specimen weight to breast volume was $0.12 \mathrm{~g} / \mathrm{mL}$ in the patients who underwent glandular tissue reshaping $(n=59)$. The corresponding values were $101.47 \mathrm{~g}$ and $0.14 \mathrm{~g} / \mathrm{mL}$, respectively, in the patients who underwent reduction oncoplasty $(n=17)$, and $82.54 \mathrm{~g}$ and $0.20 \mathrm{~g} / \mathrm{mL}$, respectively, in those treated with an LD flap $(n=32)$. Glandular tissue reshaping was mostly performed in the upper outer quadrant, and LD flap transposition was mostly performed in the lower inner quadrant. No major complications were noted. Most patients were satisfied with the aesthetic results.

Conclusions We report satisfactory outcomes of oncoplastic surgical procedures in Korean patients. The results regarding specimen weight and the tumor-to-breast ratio of Asian patients will be a helpful reference point for determining the most appropriate oncoplastic surgical technique.

Keywords Mammaplasty / Surgical flaps / Breast neoplasms

Received: 16 Apr $2017 \bullet$ Revised: 21 Sep $2017 \bullet$ Accepted: 18 Oct 2017

pISSN: 2234-6163 • elSSN: 2234-6171 • https://doi.org/10.5999/aps.2017.00836• Arch Plast Surg 2018;45:37-44
Correspondence: Dong Won Lee Department of Plastic and Reconstructive Surgery, Yonsei University College of Medicine, 50-1 Yonsei-ro, Seodaemun-gu, Seoul 03722, Korea

Tel: +82-2-2228-2215

Fax: +82-2-393-6947

E-mail:xyphoss@yuhs.ac

\section{INTRODUCTION}

In recent years, breast-conserving surgery (BCS) has become increasingly commonly performed worldwide. The prognosis after total mastectomy or BCS with adjuvant radiation therapy in patients with breast cancer was proven to be equal in cases of early-stage breast cancer [1]. The aesthetic outcome of the procedure, as well as the oncologic outcome, is important to most patients. Even though aesthetic techniques have improved, $4 \%-$ $20 \%$ of patients who undergo BCS may still be dissatisfied with the aesthetic outcome [2]. Oncoplastic surgery was first introduced in 1998 to improve both aesthetic and oncologic out- 
comes [3]. The location of the tumor and the tumor-to-breastsize ratio are known to be important factors for selecting the reconstruction method [4].

Several techniques have been developed to minimize breast deformities. The first method is the volume displacement technique, which rearranges the position of the breast parenchyma via glandular tissue reshaping, or the reduction oncoplasty technique [5]. This method is less invasive and does not result in donor site morbidity. However, the glandular tissue reshaping technique is usually applicable only to small defects, and reduction oncoplasty may require a contralateral procedure. Further, the reconstructed breasts are smaller than the preoperative breasts. The second method is the volume replacement technique, which uses autologous tissue to compensate for insufficient volume via methods using local or distant flaps, such as an intercostal artery perforator flap or a latissimus dorsi (LD) myocutaneous flap. This method is usually used for moderate-sized to large defects and can restore the preoperative breast shape. However, it may result in donor site morbidity, and requires a longer operating time.

In Western countries, where most patients have moderatesized to large breasts, the resected tumor specimen weight tends to be greater than is observed in Koreans. In the studies by Losken et al. [6] and Clough et al. [7] of Western patients, the mean tumor specimen weights after partial mastectomy were $236 \mathrm{~g}$ and $222 \mathrm{~g}$, respectively. However, in Korea, where patients tend to have moderate-sized to small breasts, the tumor specimen weight is also usually lower than that of Western patients. These race-based differences in breast characteristics necessitate a new paradigm of oncoplastic surgery that is different from the conventional Western-oriented approach. In this study, we retrospectively investigated the methods of reconstruction performed after partial mastectomy according to the weight and location of the tumor in Korean patients. We aimed to present our clinical outcomes of oncoplastic surgery, including objective data, to help determine the most appropriate method for oncoplastic surgery, especially in Asian breast cancer patients.

\section{METHODS}

\section{Patients}

A total of 108 patients who underwent BCS by an oncologic breast surgeon from 2013 to 2016 also underwent immediate oncoplastic breast surgery. We conducted preoperative counseling sessions with the patients who decided to undergo partial mastectomy by a general surgeon. We explained to the patients that at the end of partial mastectomy, we would decide whether to perform a partial reconstruction based on the judgement of the plastic surgeon and oncologic surgeon, considering the defect size, specimen weight, amount of frozen sections examined, tumor location, preoperative breast size, breast shape, and expected degree of breast deformity.

\section{Reconstruction options}

The major reconstruction options offered before surgery were volume displacement techniques (i.e., glandular tissue reshaping and reduction mammoplasty) and volume replacement techniques (i.e., LD flap transposition). In this study, those who underwent glandular tissue reshaping were classified into group 1, those who underwent LD flap transposition into group 2, and those who underwent reduction oncoplasty into group 3.

During the oncologic operation, an intraoperative frozen section analysis to establish the cancer margin and sentinel lymph node assessment were performed. If the partial mastectomy was judged sufficient to treat the breast cancer without performing total mastectomy based on the frozen section analysis results, the oncologic procedure was completed via partial mastectomy. At the end of the partial mastectomy, the plastic surgeon was requested to decide whether oncoplastic surgery was required. In general, the decision to perform oncoplastic surgery was made by the plastic surgeon after confirming the appearance of the breast after oncologic surgery. If necessary, the plastic surgeon decided whether to perform glandular tissue reshaping or LD flap transposition according to the characteristics of the defect.

Reduction oncoplasty was performed in patients with large, ptotic breasts before surgery who expressed a desire for bilateral breast reduction. Reduction mammoplasty was automatically performed on the contralateral side after partial mastectomy.

\section{Surgical techniques}

Glandular tissue reshaping involves covering the parenchymal defect by undermining the surrounding glandular tissues and suturing adjacent glandular tissues or by transposing surrounding glandular tissues to the defect. After partial mastectomy, the adjacent parenchymal tissue was separated from the chest wall fascia and skin by the plastic surgeon. Thereafter, glandular tissue advancement was attempted, and if possible, the operation was completed. However, if not, further glandular tissues were separated from the chest wall fascia and overlying skin, considering the result of the flap rotation. It was important for the flap to be elevated to avoid affecting the blood supply of the glandular flap. The elevated flap was rotated or transposed from one or both sides of the defect. After suturing the glandular tissues, we carefully checked for skin dimpling or any remaining breast deformity.

Reduction oncoplasty was performed using a Wise-pattern or 
a vertical-pattern incision depending on the tumor location. Generally, the pedicle opposite to the tumor was selected. Defects of the upper pole area were reconstructed using the inferior pedicle technique and Wise-pattern skin incisions. Defects of the lower pole area were mainly reconstructed using the vertical superomedial mastopexy technique. From the incision, tumor removal was possible from any quadrant by the oncologic surgeon. The new nipple-areolar complex location and the amount of skin to be excised were planned before surgery, using a preoperative design that considered the breast volume, ptosis degree, height, and weight. Through this method, the nipple-areolar complex and dermoglandular flap could be safely supplied with blood through a virtual pedicle (superior, medial, or inferior), and a significant amount of breast tissue and excessive skin could also be removed. Consequently, the breast shape was aesthetically improved. The ipsilateral breast was generally made $10 \%$ larger to allow for radiation fibrosis. The contralateral breast was also reduced using the same technique.

The LD flap was used as a volume replacement technique. After partial mastectomy, the thoracodorsal pedicles were identified, and the thoracodorsal nerve was ligated. The LD muscle origin and part of the lateral LD muscle were also divided. Thereafter, laparotomy sponge packing was performed on the defect to determine the required volume; the volume was then compared with that of the contralateral breast. The patient was then moved to the lateral decubitus position. The design of the LD muscle and skin paddle was drawn before creating an incision on the back. If a large-volume LD flap was required, the design was made to contain a sufficient skin paddle. After creating the incision, dissection between the skin and LD muscle was performed. Dissection was performed beneath the Scarpa fascia in large defects, and the deep fat tissues underneath the Scarpa fascia were attached to the LD muscle. If the defect size was relatively small, dissection was performed along the muscular fascia of the LD. The LD muscle was dissected in the superior direction with the thoracodorsal pedicle located underneath. After complete dissection, the LD flap supplied by the thoracodorsal pedicles was shifted to the breast defect. Meticulous bleeding control and saline irrigation were performed. Two negativedrainage tubes were inserted, and multiple quilting sutures were used at the LD donor site. Fibrin sealants (Tissel, Baxter International Inc., Utica, NY, USA) were sprayed within the cavity and manual pressure was applied to the donor site for 3 minutes to eliminate the dead space. The back incision was repaired layer by layer. After changing the patient's bed to be in the sitting position, flap insetting was performed and 2 negative-drainage tubes were inserted in the reconstructed breast. The remnant skin paddle attached to the LD flap was de-epithelized and bur- ied in the defect to fill the volume of the flap.

\section{Assessment}

The medical charts of the patients were reviewed retrospectively. Their demographic data, including age, sex, histologic cancer type, TNM stage, tumor location, chemotherapy history, and radiotherapy history, were also investigated. The preoperative breast volume of all patients was recorded using a 3-dimensinoal camera (XS-400; Axis Three, AX3 Technologies LLC., Miami, FL, USA).

Surgical data, including specimen weight and oncologic surgery method, were also recorded. The weight of the tumor specimen was measured in the operating room immediately after the surgeon removed the tumor. Data about contralateral procedures and complications during follow-up were also recorded. The tumor-to-breast ratio was calculated by dividing the weight $(\mathrm{g})$ of the tumor specimen measured in the operating room by the volume $(\mathrm{mL})$ of the breast measured by a 3-dimensional camera. The patients had postoperative regular follow-ups at 3 months, 6 months, 1 year, 2 years, and 3 years. They were asked about their satisfaction with the postoperative cosmetic outcomes during the outpatient clinic visit. On average, a postoperative aesthetic satisfaction survey was administered 12 months after surgery, usually at 8 to 9 months after the end of radiotherapy. The scores ranged from 0 to 10 , with $0-2$ indicating poor satisfaction, 2-4 not good, 4-6 fair, 6-8 good, and 8-10 excellent.

\section{RESULTS}

From 2013 to 2016, a total of 625 patients underwent partial mastectomy after consultation with a plastic surgeon, and 108 patients underwent oncoplastic surgery. The proportions of patients who underwent oncoplastic surgery are shown in Table 1. For 517 of the 625 patients (82.72\%) who underwent preoperative interviews with the plastic surgeon, surgery was terminated by the general surgeon. Of the remaining 108 patients, 59 (54.63\%) underwent glandular tissue reshaping (Fig. 1), 17

\section{Table 1. Percentages of patients who underwent various types of oncoplastic surgery}

\begin{tabular}{|lc|}
\hline Types of surgery & No. (\%) \\
\hline Oncoplastic surgery & $108(100)$ \\
Glandular tissue reshaping & $59(54.63)$ \\
Reduction oncoplasty & $17(15.74)$ \\
LD flap transposition (endoscopic-assisted & $32(29.63)$ \\
LD flap transposition) & $(14[12.96])$ \\
\hline LD, latissimus dorsi. & \\
\hline
\end{tabular}




\section{Fig. 1. Glandular tissue reshaping}

Glandular tissue reshaping in a 47-year-old woman with ductal carcinoma in situ in the right upper outer quadrant. The specimen weight was $32 \mathrm{~g}$. A total of $60.4 \mathrm{~Gy}$ of radiation was delivered to the right breast in the postoperative period. (A) Preoperative image. (B) Two-year postoperative image.
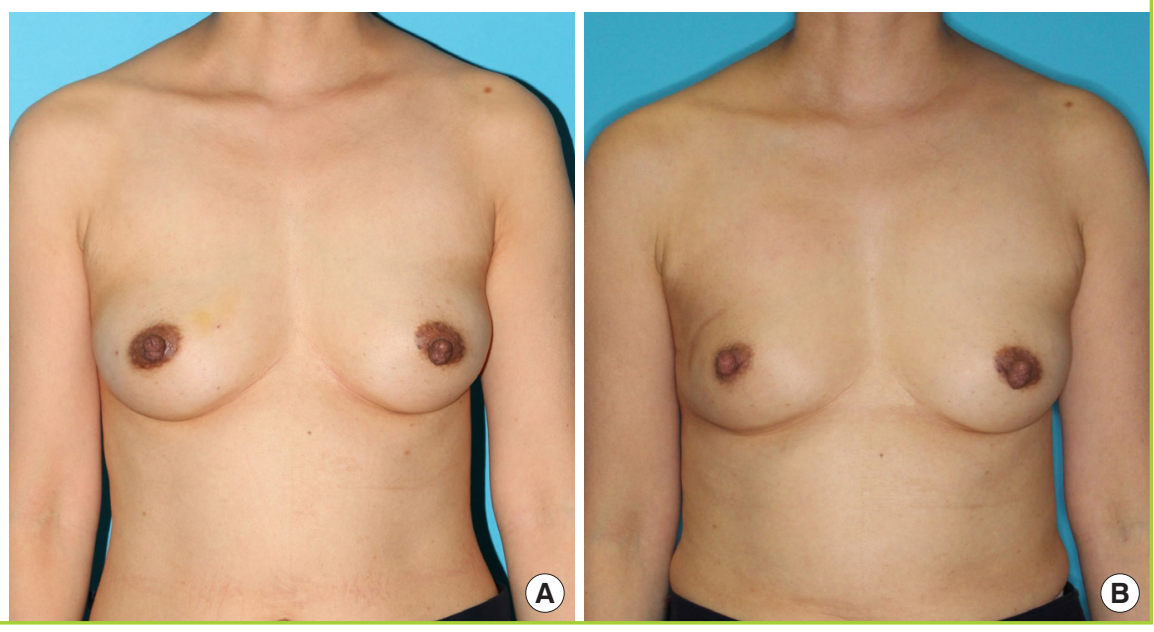

\section{Fig. 2. Reduction oncoplasty}

Reduction oncoplasty in a 49-year-old woman with invasive ductal carcinoma in the left upper outer quadrant. Tumor localization was performed preoperatively in the left breast. The specimen weight was $48 \mathrm{~g}$. Reduction mammoplasty with an inferior pedicle technique was performed. A total of $60 \mathrm{~Gy}$ of radiation was delivered to the left breast in the postoperative period. (A) Preoperative image. (B) Preoperative design.

(C) One-year postoperative image.
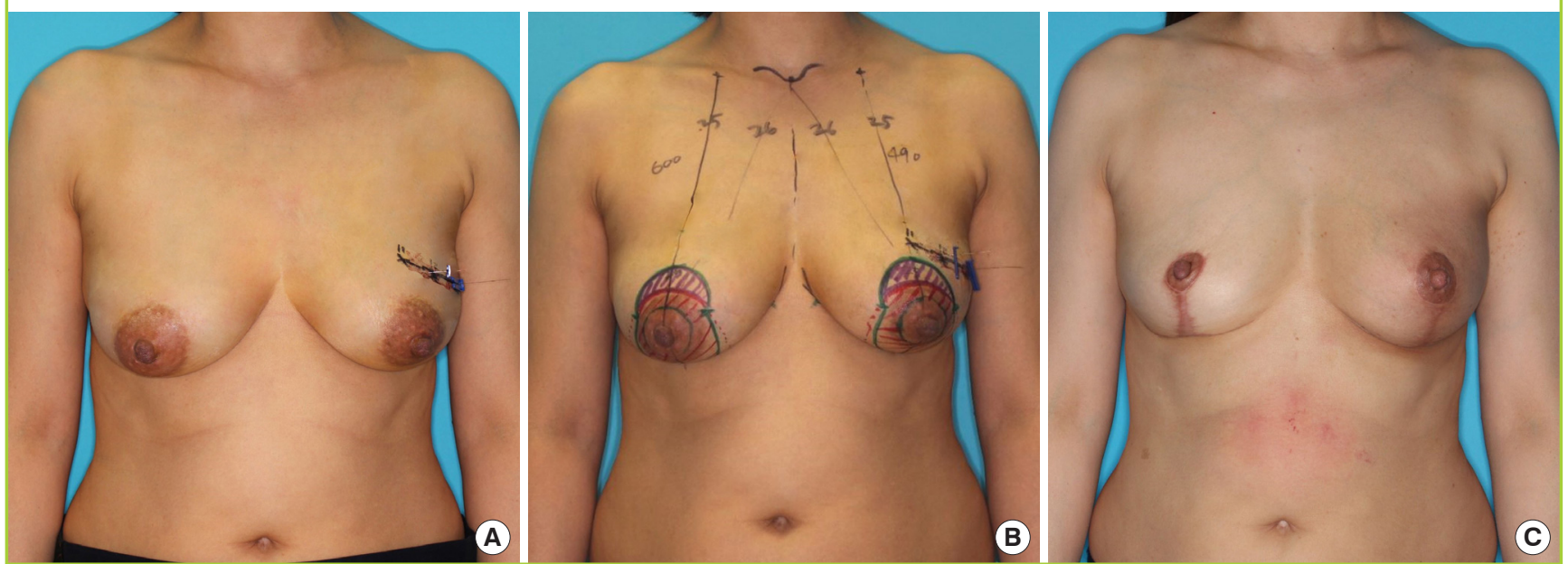

Fig. 3. Latissimus dorsi muscle flap transposition

Latissimus dorsi muscle flap transposition in a 42-year-old woman with invasive ductal carcinoma in the right lower outer quadrant. The specimen weight was $75 \mathrm{~g}$. A total of $50.05 \mathrm{~Gy}$ of radiation was delivered to the right breast in the postoperative period. (A) Preoperative image. (B) Fifteen-month postoperative image.
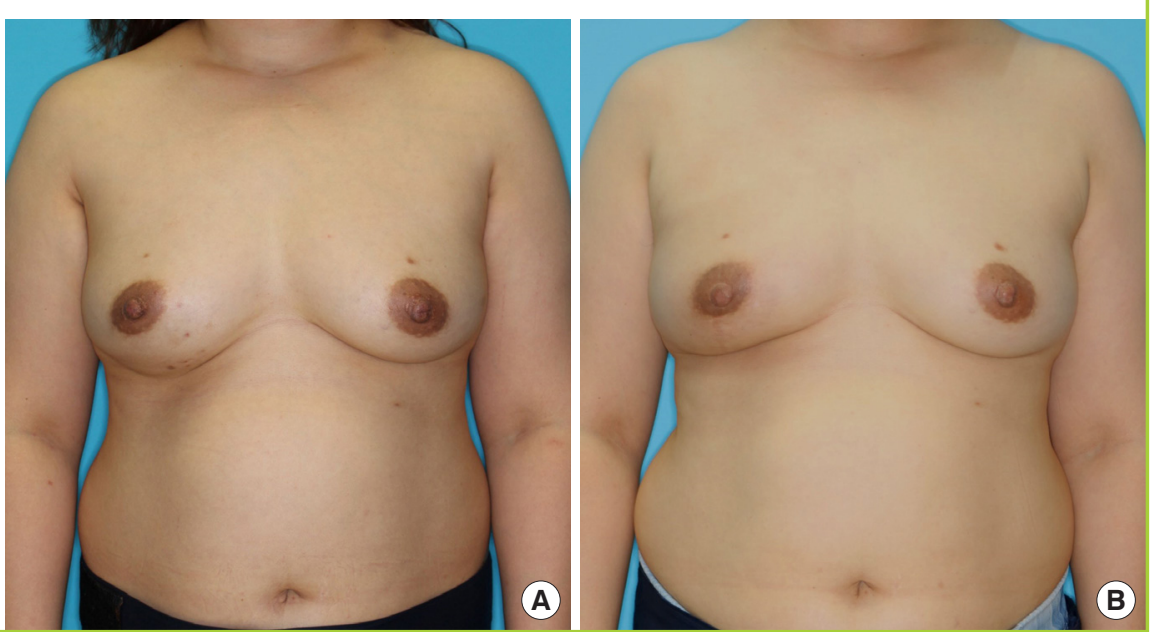
(15.74\%) underwent reduction oncoplasty (Fig. 2), and 32 (29.63\%) underwent LD flap transposition (Fig. 3). Among the 32 patients who underwent LD flap transposition, 14 (12.96\%) underwent endoscopic-assisted LD flap transposition because they needed less flap volume.

The data from the 108 patients who underwent oncoplastic surgery after BCS, including tumor location and specimen weight, were collected. The demographic data of the patients are shown in Table 2. Their age ranged from 28 to 74 years (mean, 47 years). The mean follow-up period was 14.5 months.

\section{Table 2. Characteristics of breast cancer in the patients} included in this study

\begin{tabular}{|lc|}
\hline Characteristic & Value (\%) \\
\hline Location & \\
Left & $61(56.4)$ \\
Right & $47(43.6)$ \\
Pathology & \\
Invasive ductal carcinoma & $65(60.2)$ \\
Ductal carcinoma in situ & $22(20.4)$ \\
Invasive lobular carcinoma & $6(5.5)$ \\
Lobular carcinoma in situ & $4(3.7)$ \\
Phyllodes tumor & $2(1.9)$ \\
Mucinous carcinoma & $6(5.5)$ \\
Tubular carcinoma & $2(1.9)$ \\
Invasive micropapillary carcinoma & $1(0.9)$ \\
Tumor node metastasis stage (except phyllodes tumor) & \\
O & $26(24.5)$ \\
IA & $48(45.3)$ \\
IB & $1(0.9)$ \\
IIA & $23(21.7)$ \\
IIB & $5(4.7)$ \\
IIIA & $3(2.8)$ \\
IIIB & 0 \\
IV & 0 \\
Radiotherapy & \\
Preoperative & \\
Postoperative & $1(0.9)$ \\
None & $7(92.6)$ \\
Chemotherapy & $7(6.5)$ \\
Neoadjuvant & \\
Adjuvant & $6(5.5)$ \\
None & $41(38.0)$ \\
\hline & $61(56.5)$ \\
\hline
\end{tabular}

The cancer stage of the patients ranged from 0 to IIB according to the TNM staging system of the American Joint Committee on Cancer. The most frequent histologic cancer type was invasive ductal carcinoma (65 cases, $60.2 \%$ ). Postoperative radiotherapy was performed in 100 patients $(92.6 \%)$. Neoadjuvant chemotherapy was administered to 6 patients (5.5\%), 41 patients $(38 \%)$ received adjuvant chemotherapy, and 61 patients (56.5\%) did not receive chemotherapy.

The preoperative breast volume was recorded using a 3-dimensional camera (Table 3). The mean preoperative breast volume of the 108 patients was $413 \mathrm{~mL}$, when only the side with cancer was calculated. The mean preoperative breast volume was $332 \mathrm{~mL}$ in group $1,705 \mathrm{~mL}$ in group 2, and $401 \mathrm{~mL}$ in group 3.

Tumor locations and specimen weights are shown in Table 4. The mean weight of the entire tumor specimen was $62.31 \mathrm{~g}$ (range, 4-410 g). The mean tumor specimen weight was $40.46 \mathrm{~g}$ in group 1, $101.47 \mathrm{~g}$ in group 2, and $82.54 \mathrm{~g}$ in group 3 (Table 4). The distribution of tumor locations is also shown in Table 4. When we divided the breasts into quadrants, most of the tumors $(49.1 \%)$ were located in the upper outer quadrant (quadrant 1 ) in group 1. In group 3, most tumors (31.3\%) were located in the lower inner quadrant (quadrant 4). In group 2, most tumors $(58.8 \%)$ were located in the upper outer quadrant (quadrant 1). The tumor-to-breast ratio is shown in Table 4. As described above, the ratio $(\mathrm{g} / \mathrm{mL})$ was calculated by dividing the specimen weight $(\mathrm{g})$ by the breast volume $(\mathrm{mL})$. The ratios were 0.12 in group $1,0.14$ in group 2 , and 0.20 in group 3 .

When we investigated the complications, 2 cases of minor

\section{Table 3. Preoperative volume of the breasts}

\begin{tabular}{|lc|}
\hline Group & Preoperative volume $(\mathrm{mL})$ \\
\hline Glandular tissue reshaping (group 1): 59 cases & 332 \\
Reduction oncoplasty (group 2): 17 cases & 705 \\
LD flap transposition (group 3): 32 cases & 401 \\
Total: 108 cases & 413 \\
\hline LD, latissimus dorsi. & \\
\hline
\end{tabular}

Table 4. Specimen weights and tumor location according to the surgical technique

\begin{tabular}{|c|c|c|c|c|c|c|c|c|}
\hline \multirow{2}{*}{ Surgical technique } & \multirow{2}{*}{ No. } & \multicolumn{5}{|c|}{ Location of the tumor } & \multirow{2}{*}{$\begin{array}{l}\text { Specimen } \\
\text { weight }(g)\end{array}$} & \multirow{2}{*}{$\begin{array}{l}\text { Tumor to breas } \\
\text { ratio }(\mathrm{g} / \mathrm{mL})\end{array}$} \\
\hline & & U00 (no.1) & UIO (no.2) & LOQ (no.3) & LIQ (no.4) & Nipple & & \\
\hline Glandular tissue reshaping (group 1) & 59 & $29(49.1)$ & $20(33.9)$ & $4(6.8)$ & $6(10.2)$ & 0 & 40.06 & 0.12 \\
\hline Reduction oncoplasty (group 2) & 17 & $10(58.8)$ & $4(23.5)$ & $2(11.8)$ & $1(5.9)$ & 0 & 101.47 & 0.14 \\
\hline LD flap transposition (group 3) & 32 & $9(28.13)$ & $5(15.6)$ & $7(21.9)$ & $10(31.3)$ & $1(3.1)$ & 82.54 & 0.20 \\
\hline Total & 108 & $48(44.4)$ & $29(26.9)$ & $13(12.0)$ & $17(15.7)$ & $1(0.9)$ & 62.31 & - \\
\hline
\end{tabular}


wound breakdown (11.7\%) were noted in group 2, which were treated conservatively. Six cases of seroma at the donor site $(18.7 \%)$ were noted in group 3 , and were cured by repetitive needle aspirations during outpatient follow-up visits. All seromas resolved within 6 weeks after surgery. Complications such as flap necrosis and wound infection did not occur. During the follow-up period, contralateral procedures or revisional procedures were not performed in any cases. The mean postoperative degree of aesthetic satisfaction was 8.3 in group 1, 8.7 in group 2, and 8.1 in group 3 . This indicates that most patients were satisfied with their results.

\section{DISCUSSION}

Previous studies have shown that there is no difference in the outcomes between BCS with radiation and mastectomy alone in early-stage breast cancer $[1,8]$. Some studies have even reported that BCS with radiation appeared to yield an equivalent outcome or a better survival rate than total mastectomy alone $[9,10]$. However, according to a previous study, the aesthetic results were unsatisfactory in $30 \%$ of patients who underwent BCS that removed $>20 \%$ of the original breast volume [11]. This indicates that patients who underwent the discouraging experience of breast cancer surgery often underwent another discouraging event: having an unwanted postoperative breast shape. To relieve this psychiatric burden, oncoplastic surgery is being increasingly performed in accordance with the increasing number of BCS procedures. Oncoplastic surgery, as well as BCS, is another important treatment method for patients with breast cancer.

Conceptually, oncoplastic surgery involves volume displacement and replacement techniques. Considering the characteristics of Asians, who usually have small to moderate-sized breasts unlike Westerners, Yang et al. [12] put forth an algorithm that recommends volume displacement techniques for excised volumes $<50 \mathrm{~g}$; thoracoepigastric, intercostal artery perforator, lateral thoracodorsal, and thoracodorsal artery perforator flaps for excised volumes of 50-150 g according to the tumor location; and LD flaps for excised volumes $>150 \mathrm{~g}$. Similarly, glandular tissue reshaping, LD flap transposition, and reduction oncoplasty were performed as oncoplastic procedures are our institution based on patients' characteristics.

Glandular tissue reshaping was performed with an average tumor specimen weight of $40 \mathrm{~g}$, mostly in the upper outer quadrant. When the tumor is located in the upper breast, especially in the upper outer quadrant, and the defect is not large (tumor weight $<60 \mathrm{~g}$ ), glandular tissue reshaping can be performed successfully because the surrounding breast tissues are lax and sufficient for glandular tissue reshaping. Likewise, local fasciocutaneous flaps can be used to cover small lateral defects ( $<10 \%$ of the breast size), according to Clough et al. [13]. In a previous study, a local fasciocutaneous flap was required because the lesion was removed, including the skin; however, glandular flap reshaping was sufficient in our study because the skin was usually not removed.

In cases of small lower pole defects, we were also able to treat the defect well, without deformation, by glandular tissue reshaping. The glandular tissue reshaping method is easy to perform in upper pole defects, but with a detailed surgical plan and if an appropriate defect is selected, it can also be used to treat small lower pole defects. During the follow-up period, 2 cases of nippleareolar complex deformity and 3 cases of mild breast skin depression were observed after radiotherapy. After consultation with the patients, we decided to perform conservative treatment and regular follow-up. Except for these cases, nearly symmetric shapes of the breast were maintained.

Reduction oncoplasty can be an effective surgical procedure for patients who have large, ptotic breasts before surgery and have considered breast reduction. In addition, since the plastic surgeon resects an additional tissue margin, this method has advantages over other surgical methods in terms of oncologic outcomes. From an aesthetic viewpoint, patient satisfaction is high because these patients also achieve a more beautiful and symmetrical breast than they had before surgery; further, chronic shoulder and neck pain owing to large breasts can be relieved after reduction oncoplasty.

In LD flap surgery, the thoracodorsal artery is used as a pedicle, and the required amount of LD muscle is elevated and transposed to the defect site. If a significant amount of the breast skin is removed, the skin paddle of the $\mathrm{LD}$ flap can fill the breast defect. The disadvantage of this technique is the presence of a transverse scar on the back and an elevated risk of seroma at the donor site; however, this technique is useful when the volume deficit is relatively large and the breast shape might not be effectively recovered using volume displacement techniques, especially in small to moderate-sized breasts. In this study, the mean specimen weight in cases of LD flap transposition was $82.54 \mathrm{~g}$, and the most frequent tumor location was the lower inner quadrant. The LD flap is a common flap option for lateral, central, and even medial defects [14]. This flap can contain not only the LD muscle, but also subcutaneous tissue and skin; thus, it can cover large-sized defects well. A denervated and radiated LD muscle will undergo postoperative atrophy [15]. We tried to provide the required volume mainly by regulating the amount of deepithelized dermal tissues in the LD flap, as such tissue undergoes less atrophy than the LD muscle. According to Co- 
chrane et al. [16], patient satisfaction with cosmetic outcomes is relatively low when the breast cancer is located in the medial area of the breast. In the lower inner quadrant, where deformation is likely to occur when the volume is inadequate, the $\mathrm{LD}$ flap method was a suitable option to reconstruct the breast similarly to the preoperative breast.

Recently, endoscopic-assisted LD flap transposition has been introduced and used; this technique leaves less scarring on the back than conventional LD flap transposition [17]. Considering the tumor location, especially in the upper and lower outer quadrants, endoscopic-assisted LD flap transposition is beneficial for patients who want less scarring [18]. In this study, endoscopic-assisted LD flap transposition was mainly performed for tumors in the upper outer quadrant; 7 of the 14 endoscopic-assisted LD flap transposition cases had a tumor located in the upper outer quadrant. Due to the limited accessibility and difficulty of harvesting, the harvested LD volume is usually slightly less than that of conventional LD flap procedures. A mean specimen weight of $74.82 \mathrm{~g}$ was obtained when surgery was performed with an endoscopic-assisted LD flap in this study.

The incidence of seroma (18.7\%) was low in comparison to the complication rates of other studies. In other studies, seroma incidence has been reported to be $6 \%$ to $80 \%$ in cases of autologous $\mathrm{LD}$ flap reconstruction and $\mathrm{LD}$-implant reconstruction [19]. As described in a previous study [20], a dramatic decrease in the seroma rate was confirmed when fibrin sealant and quilting sutures were used simultaneously. According to Pogson et al. [21], delaying formal shoulder physiotherapy exercises may reduce the total drainage volume. We used fibrin sealants, quilting sutures, and shoulder immobilization during the postoperative hospitalization period to minimize the seroma rate.

Conventionally, the tumor-to-breast-size ratio is believed to be a key factor that determines the decision to perform oncoplastic surgery after BCS. According to previous studies, poorer cosmetic scores were noted in patients who had parenchymal resections of $>70-100 \mathrm{~cm}^{3}$ or when the ratio of specimen weight to breast volume exceeded $10 \%[20,22,23]$. In our study, the tumor-to-breast-size ratio in group 1 was $12 \%$, while it was $20.4 \%$ in group 3. Altogether, patients who showed ratios $>10 \%$ would need to undergo oncoplastic surgery using a volume displacement technique, while those who showed ratios $>20 \%$ would need to undergo oncoplastic surgery using a volume replacement technique.

Compared with the results of previous studies of oncoplastic surgery in Koreans, we found that the mean specimen weights of each group of patients of this study were much smaller than those reported in previous studies [12,24]. In another study, the mean specimen weight of LD flaps was $182.6 \mathrm{~g}$, and LD flaps were recommended for cases with a specimen weight $>150 \mathrm{~g}$ [12]. However, our study showed that the average specimen weight of LD flap patients was $82.54 \mathrm{~g}$. In previous studies, LD flap surgery was mainly used for only large defects, while moderate defects were covered by lateral thoracodorsal flaps, intercostal artery perforator flaps, thoracoepigastric flaps, and thoracodorsal artery perforator flaps.

Since the volume of the LD flap was determined by controlling the amount of dermal tissue, the flap volume was precisely adjusted in this study according to the degree of severity. Therefore, we were able to effectively reconstruct moderate-sized defects, as well as large defects.

In conclusion, the glandular tissue reshaping method was suitable for small defects with a mean specimen weight of $40.06 \mathrm{~g}$ and a ratio of tumor weight to breast volume of $12 \%$. It was easy to perform the operation when the defect was located at the upper pole. LD flap surgery was performed with an average specimen weight of $82.54 \mathrm{~g}$ and a ratio of tumor weight to breast volume of $20 \%$. For defects in the lower inner quadrant, reconstruction using an LD flap may be more necessary, and LD flaps can be effectively used for reconstructing defects at all sites of the breast.

For plastic surgeons who reconstruct breasts, considering the specimen weight and tumor location is very important for reconstructing breasts naturally and symmetrically. We expect that this study will be helpful for determining the optimal method of oncoplastic surgery.

There were a few limitations of this study. First, we did not consider the frozen section specimen weight. Sometimes, frozen section analysis is performed 2 or even 3 times when the resection margin is proven positive for cancer. However, we could not calculate the frozen section specimen weight, so we only considered the permanent tumor specimen weight. If the frozen section margin weight had been taken into account, the average specimen weight would have also increased. Second, the study had a relatively small sample size. More than 100 patients were included in this study; however, after sorting the data according to the tumor location and surgical method, each group had a relatively small sample size. Because of the small sample size of each group, we had difficulty in performing statistical analyses. If larger sample sizes had been included in this study, more obvious differences could have been noted and a more reliable statistical analysis could have been performed. Third, the mean follow-up period was short and the aesthetic satisfaction survey was administered not long after the completion of radiation therapy. The surveys were mainly completed when patients visited our outpatient clinic 12 months postoperatively. Changes in breast shape and volume may occur more than 1 year after radia- 
tion therapy. If we had conducted satisfaction surveys 2 years after surgery, our results would have been stronger.

\section{CONFLICT OF INTEREST}

No potential conflict of interest relevant to this article was reported.

\section{PATIENT CONSENT}

The patient provided written informed consent for the publication and the use of their images.

\section{REFERENCES}

1. NIH consensus conference. Treatment of early-stage breast cancer. JAMA 1991;265:391-5.

2. Al-Ghazal SK, Blamey RW. Cosmetic assessment of breastconserving surgery for primary breast cancer. Breast 1999;8:1628.

3. Clough KB, Cuminet J, Fitoussi A, et al. Cosmetic sequelae after conservative treatment for breast cancer: classification and results of surgical correction. Ann Plast Surg 1998;41:47181.

4. Serra-Renom JM, Serra-Mestre JM, Martinez L, et al. Endoscopic reconstruction of partial mastectomy defects using latissimus dorsi muscle flap without causing scars on the back. Aesthetic Plast Surg 2013;37:941-9.

5. Anderson BO, Masetti R, Silverstein MJ. Oncoplastic approaches to partial mastectomy: an overview of volume-displacement techniques. Lancet Oncol 2005;6:145-57.

6. Losken A, Styblo TM, Carlson GW, et al. Management algorithm and outcome evaluation of partial mastectomy defects treated using reduction or mastopexy techniques. Ann Plast Surg 2007;59:235-42.

7. Clough KB, Lewis JS, Couturaud B, et al. Oncoplastic techniques allow extensive resections for breast-conserving therapy of breast carcinomas. Ann Surg 2003;237:26-34.

8. Fisher B, Anderson S, Bryant J, et al. Twenty-year follow-up of a randomized trial comparing total mastectomy, lumpectomy, and lumpectomy plus irradiation for the treatment of invasive breast cancer. N Engl J Med 2002;347:1233-41.

9. Arriagada R, Le MG, Rochard F, et al. Conservative treatment versus mastectomy in early breast cancer: patterns of failure with 15 years of follow-up data. Institut Gustave-Roussy Breast Cancer Group. J Clin Oncol 1996;14:1558-64.

10. Lichter AS, Lippman ME, Danforth DN Jr, et al. Mastecto- my versus breast-conserving therapy in the treatment of stage I and II carcinoma of the breast: a randomized trial at the National Cancer Institute. J Clin Oncol 1992;10:976-83.

11. Urban C, Lima R, Schunemann E, et al. Oncoplastic principles in breast conserving surgery. Breast 2011;20 Suppl 3:S925.

12. Yang JD, Kim MC, Lee JW, et al. Usefulness of oncoplastic volume replacement techniques after breast conserving surgery in small to moderate-sized breasts. Arch Plast Surg 2012;39:489-96.

13. Clough KB, Kroll SS, Audretsch W. An approach to the repair of partial mastectomy defects. Plast Reconstr Surg 1999;104:40920.

14. Losken A, Schaefer TG, Carlson GW, et al. Immediate endoscopic latissimus dorsi flap: risk or benefit in reconstructing partial mastectomy defects. Ann Plast Surg 2004;53:1-5.

15. Losken A, Hamdi M. Partial breast reconstruction: current perspectives. Plast Reconstr Surg 2009;124:722-36.

16. Cochrane RA, Valasiadou P, Wilson AR, et al. Cosmesis and satisfaction after breast-conserving surgery correlates with the percentage of breast volume excised. Br J Surg 2003;90:15059.

17. Missana MC, Pomel C. Endoscopic latissimus dorsi flap harvesting. Am J Surg 2007; 194:164-9.

18. Yang CE, Roh TS, Yun IS, et al. Immediate partial breast reconstruction with endoscopic latissimus dorsi muscle flap harvest. Arch Plast Surg 2014;41:513-9.

19. Daltrey I, Thomson H, Hussien M, et al. Randomized clinical trial of the effect of quilting latissimus dorsi flap donor site on seroma formation. Br J Surg 2006;93:825-30.

20. Shin IS, Lee DW, Lew DH. Efficacy of quilting sutures and fibrin sealant together for prevention of seroma in extended latissimus dorsi flap donor sites. Arch Plast Surg 2012;39:50913.

21. Pogson CJ, Adwani A, Ebbs SR. Seroma following breast cancer surgery. Eur J Surg Oncol 2003;29:711-7.

22. Mills JM, Schultz DJ, Solin LJ. Preservation of cosmesis with low complication risk after conservative surgery and radiotherapy for ductal carcinoma in situ of the breast. Int J Radiat Oncol Biol Phys 1997;39:637-41.

23. Taylor ME, Perez CA, Halverson KJ, et al. Factors influencing cosmetic results after conservation therapy for breast cancer. Int J Radiat Oncol Biol Phys 1995;31:753-64.

24. Lee JW, Kim MC, Park HY, et al. Oncoplastic volume replacement techniques according to the excised volume and tumor location in small- to moderate-sized breasts. Gland Surg 2014;3:14-21. 